\title{
Enterourachal Fistula as an Initial Presentation in Crohn Disease
}

\author{
Senthilkumar Sankararaman, Ramy Sabe, Thomas J. Sferra, and Ali Salar Khalili \\ Division of Pediatric Gastroenterology, Department of Pediatrics, UH Rainbow Babies \& Children's Hospital, Case \\ Western Reserve University School of Medicine, Cleveland, $\mathrm{OH}$, United States
}

\begin{abstract}
Crohn disease has a wide spectrum of clinical presentations and rarely can present with complications such as a bowel stricture or fistula. In this case report, we describe a 17 -year-old male who presented with a history of recurrent anterior abdominal wall abscesses and dysuria. He was diagnosed with Crohn disease and also found to have a fistulous communication between the terminal ileum and a patent urachus. An ileocecectomy with primary anastomosis and complete resection of the abscess cavity was performed. He is on azathioprine for maintenance therapy and currently in remission. Clinicians should have a high index of suspicion for this complication in Crohn disease patients presenting with symptoms suggestive of urachal anomalies such as suprapubic abdominal pain, dysuria, umbilical discharge, and periumbilical mass.
\end{abstract}

Key Words: Crohn disease, Inflammatory bowel diseases, Intestinal fistula, Urachus

\section{INTRODUCTION}

Crohn disease (CD) can present as a disease complication such as a bowel stricture or fistula. Fistula may occur between adjacent bowel loops or between a bowel loop and an adjacent organ. In this report, we present a patient who was admitted with a history of recurrent anterior abdominal wall abscesses and dysuria. He was found to have CD with a fistulous communication between the terminal ileum (TI) and a patent urachus resulting in recurrent abdominal wall abscesses.

\section{CASE REPORT}

A 17-year-old male presented with a one-week history of abdominal pain and dysuria. He described the abdominal pain as sharp and localized to the infraumbilical and right inguinal regions. He reported a decrease in appetite. He lost approximately $15 \mathrm{~kg}$ over the preceding five months. He had no fever. He had normal bowel movements without blood or mucus. His past medical history was significant for recurrent anterior abdominal wall abscesses. The initial abscess occurred five months prior and he was

Received : December 7, 2017, Revised : April 5, 2018, Accepted : April 7, 2018

Corresponding author: Ali Salar Khalili, Division of Pediatric Gastroenterology, Department of Pediatrics, UH Rainbow Babies \& Children's Hospital, Suite 737, MS RBC 6004, 11100 Euclid Avenue, Cleveland OH 44106, United States. Tel: +1-216-844-1765, Fax: +1-216-844-8750, E-mail: Ali.Khalili@UHhospitals.org

Copyright (c) 2019 by The Korean Society of Pediatric Gastroenterology, Hepatology and Nutrition

This is an open-access article distributed under the terms of the Creative Commons Attribution Non-Commercial License (http://creativecommons.org/licenses/by-nc/4.0/) which permits unrestricted non-commercial use, distribution, and reproduction in any medium, provided the original work is properly cited. 
treated with intravenous (IV) antibiotics at an outside facility. At that institution, computed tomography (CT) of the abdomen was reported as demonstrating significant bowel wall thickening of the TI with adjacent inflammatory stranding. After the antibiotic course was completed, a repeat CT showed resolution of the abdominal wall abscess but persistence of the bowel wall abnormality. Two months later, he developed a second episode of abdominal wall abscess formation that required percutaneous surgical drainage and antibiotic therapy (amoxicillin and metronidazole). Aspirated serous fluid contained few leukocytes and was sterile. After this second episode, he underwent esophagogastroduodenoscopy (EGD) and colonoscopy at the outside institution. The EGD was normal and colonoscopy revealed erythema with ulcerations in the TI. Histopathology confirmed severe chronic inflammation of TI. He was prescribed an oral 5-aminosalicylic acid for inflammatory bowel disease. He was not compliant with taking this medication. His abdominal pain slightly improved for a brief period of time. A follow-up colonoscopy was planned at the other institution but he was admitted to our hospital with the current presenting symptoms.

On admission, his vital signs were within normal limits. Physical examination revealed a soft, nondistended abdomen with mild tenderness in the infraumbilical and right inguinal regions. A small area of erythema and induration, approximately $2 \mathrm{~cm}$ in diameter, was present just inferior to the umbilicus. There was minimal guarding but no rigidity or rebound. No umbilical discharge was noted. Laboratory evaluation demonstrated elevated inflammatory markers and normal hemoglobin, platelet count, albumin, and urinalysis. The CT of the abdomen revealed a rim enhancing ovoid shaped fluid collection in the midline of the suprapubic region (just below the umbilicus) measuring $10.5 \times 3 \times 2.5$ $\mathrm{cm}$ (Fig. 1A). This abscess cavity contained multiple small foci of gas tracking from the umbilicus to the superior-ventral aspect of the urinary bladder wall. Contiguous with the ovoid fluid collection and just lateral to the right rectus abdominis muscle, there was a smaller abscess approximately $1.5 \mathrm{~cm}$ in diameter. The $\mathrm{CT}$ also revealed significant thickening of the wall of the distal ileum that was in close contiguity with the urachal remnant suggesting the presence of an enterourachal fistula (Fig. 1B). The prior CT performed at the outside hospital at the time of initial presentation was reviewed at our institution and revealed a patent urachus at the same location of the current ovoid shaped abscess (Fig. IC). He was started on IV piperacillin-tazobactam. He underwent EGD and colonoscopy. EGD was normal. Colonoscopy revealed severe erythema and ulceration in the TI and colon appeared normal. Biopsies from TI revealed chronic severe ileitis with architectural distortion. The colonic biopsies were normal. Magnetic resonance enterography (MRE) confirmed the CT scan findings and clearly demonstrated a fistula from the TI to the urachal remnant abscess (Fig. 1D). Quantiferon-TB gold test was negative and chest radiograph was normal. He was diagnosed with fistulizing CD based on the endoscopic, histologic, and MRE findings. Induction therapy for CD with prednisone was initiated. Percutaneous drainage of the abscess was performed. The aspirated fluid was purulent with neutrophilic predominance and no organism was cultured. He completed two weeks of IV piperacillin-tazobactam and two weeks of oral ciprofloxacin and metronidazole therapy. At the completion of the antibiotic course, a CT scan with sinogram (contrast administered through the drainage catheter) showed no residual fluid collection and no significant filling of the abscess cavity with contrast. The drainage catheter was removed. Repeat MRE showed interval improvement of the TI thickening, complete resolution of the anterior abdominal abscesses, and a blind-ended fistula from the TI without communication to the urachal remnant. His inflammatory markers slowly normalized. Prednisone was gradually weaned and he was started on azathioprine for maintenance therapy. He was referred for a colorectal surgical consultation and underwent laparoscopic exploration of the abdomen. The old abscess cavity was identified and noted to be tightly adherent to the anterior ab- 

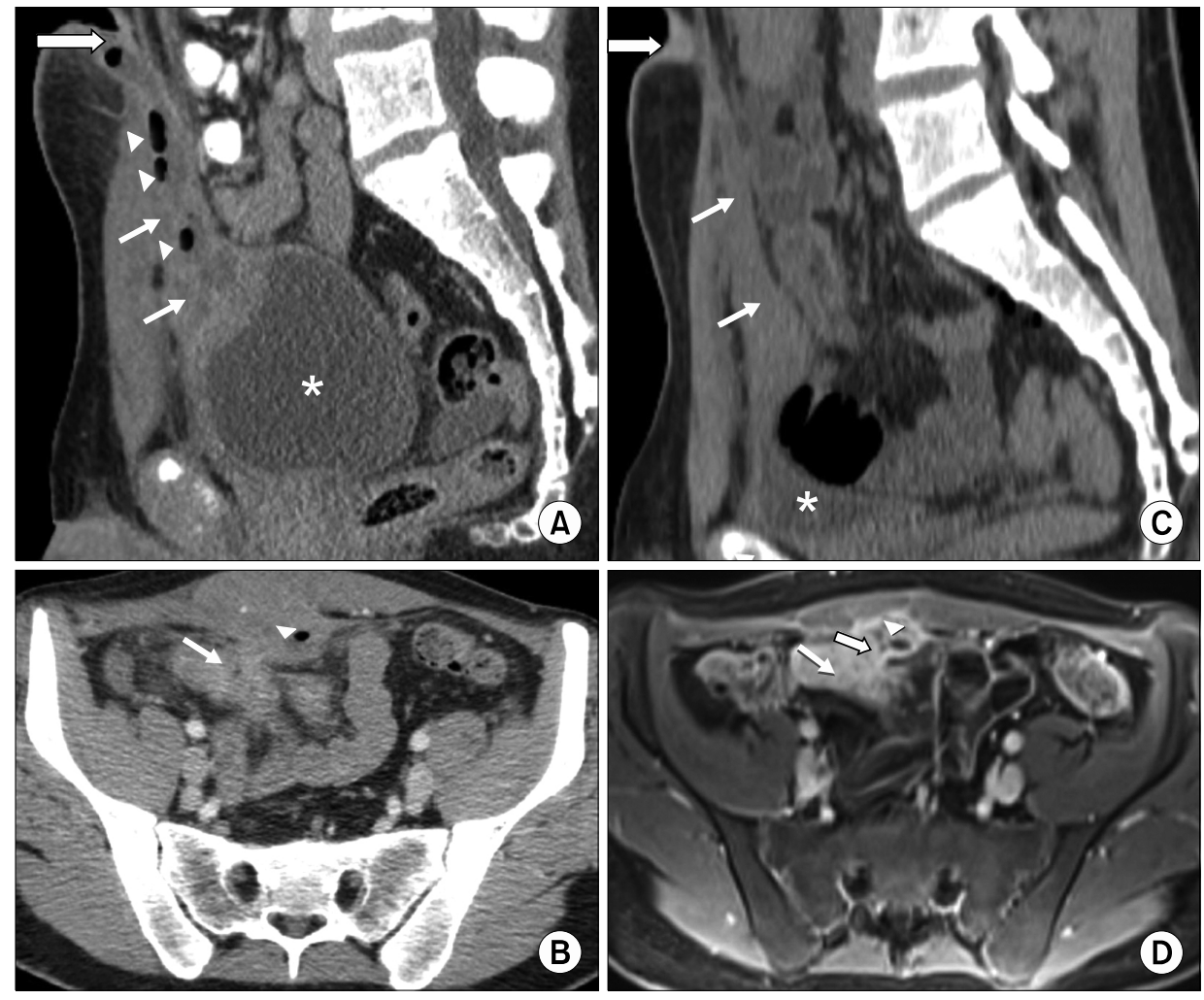

Fig. 1. (A) Sagittal computed tomography (CT) image of the abdomen and pelvis demonstrating the urachus (white arrows) which connects the urinary bladder (asterisk) and umbilicus (white arrow with black outline). The urachal remnant is thickened and distended with fluid and gas pockets (white arrow heads) consistent with an abscess. Also, the inflammatory stranding of the anterior abdominal wall is evident. (B) Axial CT image of the pelvis demonstrating the thickened wall of the distal ileum (white arrow) contiguous with the urachal remnant (white arrow head). This is suggestive of a fistula between these two structures. The urachal remnant appears edematous with a gas pocket which is consistent with an abscess and the edema extends to involve the adjacent abdominal wall. (C) Sagittal CT image of the abdomen and pelvis taken at an outside facility before the enterourachal fistula formation ( 5 months prior to this presentation). Urachal remnant (white arrows) extends from the anterior dome of the urinary bladder (asterisk) to the umbilicus (white arrow with black outline). (D) Axial magnetic resonance enterography with volumetric interpolated breath-hold examination image of the pelvis 3 minutes post contrast administration. Fistulous tract between urachal remnant and distal ileum clearly identified (white arrow with black outline). Marked thickening of the distal ileal wall (white arrow) and urachal remnant wall with some fluid remaining in the urachal remnant (white arrow head) consistent with an abscess.

dominal wall and in close proximity to the inflamed TI. Ileocecectomy with primary anastomosis and complete resection of the abscess cavity was performed. The enterourachal fistula could not be identified and likely obliterated with the medical management. Resection of the urachal remnant could not be simultaneously done due to dense adhesions. Histopathology of the resected specimen confirmed active CD. A repeat colonoscopy ten months after the surgery revealed the $C D$ in remission. He had no recurrence of abscesses during the follow up. A complete resection of the urachal remnant has been planned.

\section{DISCUSSION}

Fistula formation is a well-known complication of $\mathrm{CD}$ resulting from the transmural inflammation characteristic of this disease. Fistula may form between adjacent bowel loops and any adjacent structure including the retroperitoneum, urinary bladder, vagina, and skin. A fistulous communication be- 
tween urachus and inflamed bowel from CD is exceedingly rare and only a few cases are reported [1-12]. Due to the rarity and nonspecific presentation, the diagnosis of enterourachal fistula can be delayed [9].

We did a systematic literature search of MEDLINE and Embase databases using the following terms Crohn's disease, inflammatory bowel disease, IBD, urachus, and urachal. We identified descriptions of twelve cases of $\mathrm{CD}$ with an associated urachal complication (Table 1) [1-12]. In non-English language journal articles, only abstracts were reviewed and included. We also found two retrospective chart reviews and one case series which described four additional patients with $\mathrm{CD}$ and urachal anomalies [13-15]. These four patients are not included in the table 1 due to lack of clinical details [13-15]. Solem et al. [13] reviewed $78 \mathrm{CD}$ patients and found two patients had fistula between the ileum and urachus. Ishii et al. [15] reviewed 1,551 CD patients and one patient was identified with urachal abscess and enterourachal cutaneous fistula. This patient underwent resection of the urachus with resection of inflamed intestine, and partial cystectomy. Paşalega et al. [14] reported a patient with CD and infected urachal cyst. Including the patient reported here, the total number of $\mathrm{CD}$ patients with urachal anomalies reported in the literature since the publication of first case in the year 1980 is seventeen [1-15].

The age range of these patients was between 11 to 31 years and the median age was 19 years [1-12]. The male-to-female ratio was almost equal. Abdominal pain was the most common presenting symptom ( 10 of 13 patients). Umbilical discharge ( 6 of 13) and urinary symptoms such as dysuria, fecaluria, increased frequency (4 of 13) were also described. Other presenting symptoms included diarrhea, weight loss, and fever. The ileocecal region was involved in most patients. The urachal abnormalities described include enterourachal fistula and infected urachal cyst [1-15]. Radiological imaging modalities such as CT and magnetic resonance imaging (MRI) were helpful in diagnosing urachal anomalies and also helpful in the suspicion of a fistulous complication. Either laparoscopy or laparotomy was useful in confirming enterourachal fistula. Most patients were successfully managed by urachal resection with partial cystectomy along with partial resection of the inflamed bowel.

Similar to the patient described here, the enterourachal fistula could be the initial presentation of $\mathrm{CD}$ and this causes a diagnostic challenge [12]. The patient described by Tsukui et al. [12] had a one year history of diarrhea, weight loss, polyuria, and the sensation of residual urine. She was initially diagnosed as having a urinary tract infection and managed conservatively. As her symptoms did not improve, she was admitted for further management and found to have a ceco-urachal fistula. On the contrary, in some patients, the urachal complication alone was diagnosed initially and fistulizing $\mathrm{CD}$ diagnosis was missed resulting in prolongation of symptoms. Sugiyama et al. [4] reported a 19-year-old woman with abdominal pain and recurrent fever for six months. Imaging revealed an urachal abscess and she underwent total urachal resection with partial resection of the bladder. A month later she developed fecaluria and dysuria. She was conservatively managed and her symptoms returned 3 months later. CT demonstrated a purulent collection in the peritoneal cavity. Cystoscopy and barium contrast study of the small bowel showed an enterovesical fistula. She underwent partial resection of the ileum and bladder. She was subsequently found to have longitudinal ulcers in the ileum with granulomas confirming CD.

Infected urachal cyst or sinus without a clearly identifiable enterourachal fistula could be another urachal complication in $\mathrm{CD}$. In two patients described by Weitten et al. [7] and O'Brien et al. [10], septic urachal cysts were noted without an identifiable fistulous tract. The infection likely developed from a microperforation of bowel wall located adjacent to urachus resulting in local bacterial translocation. Paşalega et al. [14] also reported a patient with CD and infected urachal cyst. Keir et al. [5] described a case of infected urachal sinus with granulomatous appendicitis. Resection of the in- 


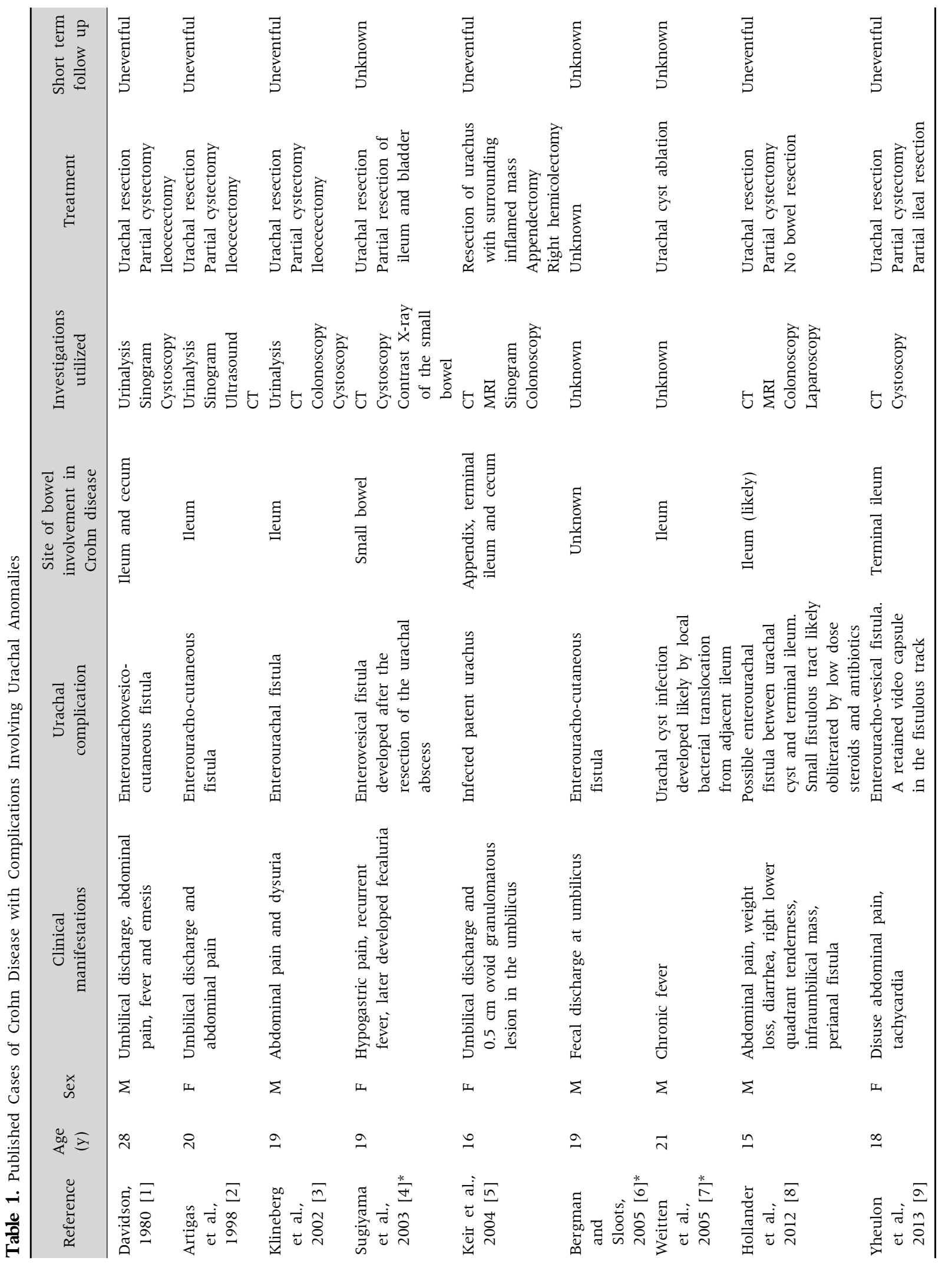




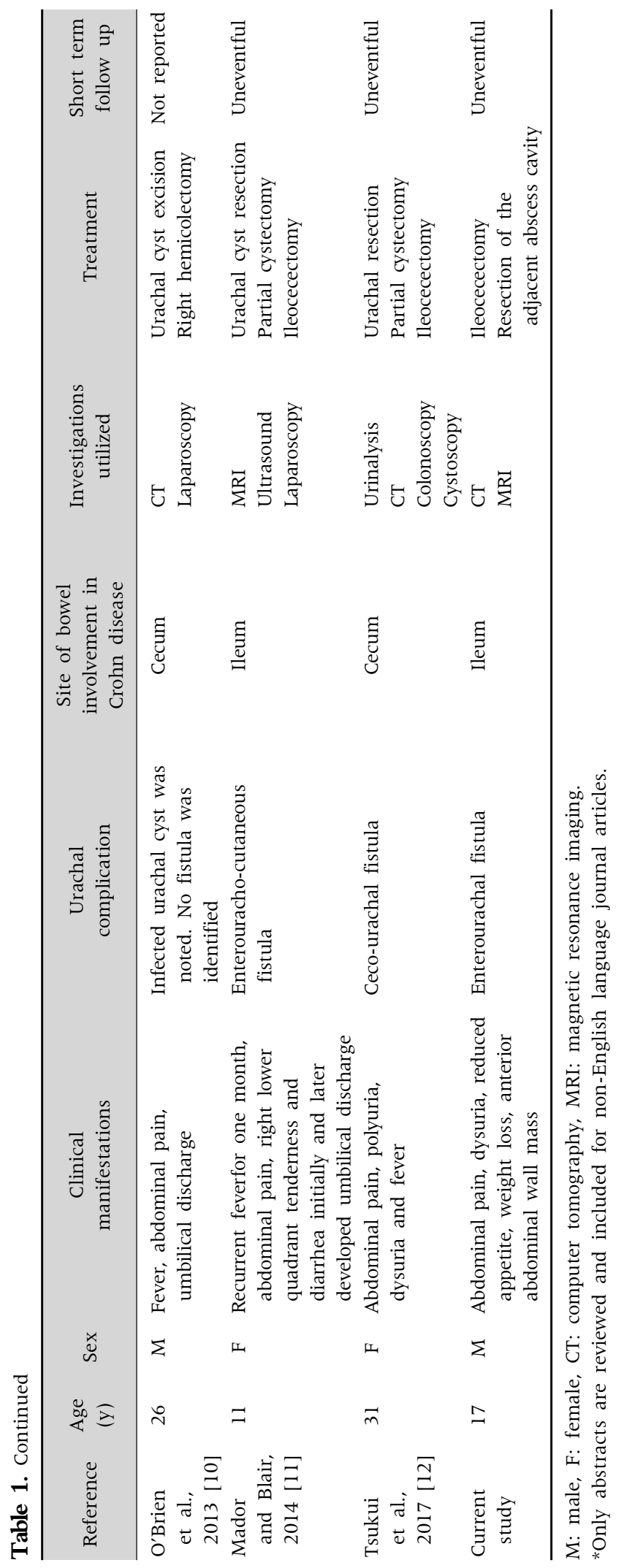


flammatory mass involving the urachus with appendectomy was initially performed. A year later she developed wound discharge. A sinogram showed small bowel stricture and also a fistula between bowel segments. Colonoscopies with biopsies were consistent with $\mathrm{CD}$ and she was started on azathioprine. Here, the authors proposed that urachal infection is likely secondary to intra-abdominal sepsis from CD.

Enterourachal fistula sometimes could complicate the course of $\mathrm{CD}$ management. In the patient described by Yheulon et al. [9], the diagnosis of CD was initially doubted after a normal video capsule endoscopy and the medications were discontinued. She presented later at a different institution with abdominal pain, tachycardia, and leukocytosis. CT scan showed a metallic object, likely a retained video capsule, within the bowel in the left lower quadrant and a 4 -cm phlegmon in the right lower quadrant adjacent to the bladder. She was conservatively managed with IV steroids and antibiotics. Repeat CT showed no metallic object in the abdomen indicating the possible spontaneous passage of the video capsule. She underwent laparotomy as the abscess increased in size despite continued medical therapy. Active $\mathrm{CD}$ in the TI and a $5 \mathrm{~cm}$ infected urachal cyst with vesico-urachal and enteral urachal fistulas were noted during laparotomy. The authors stated that the video capsule was likely retained at the site of enterourachovesical fistula.

Our case report is important in many aspects. As this combination of $\mathrm{CD}$ with urachal complication is rare, either the diagnosis of $\mathrm{CD}$ or the diagnosis of urachal complications can be easily missed resulting in treatment delay. In our patient, the urachal abnormality was not diagnosed at the other institution and patient was not compliant with the prescribed IBD management. He had recurrence of symptoms prior to presenting to us. The combination of presenting symptoms (i.e., dysuria, infraumbilical pain), recurrent anterior abdominal wall abscesses, terminal ileitis, and typical radiological findings led to the diagnosis of CD fistulizing to the urachal remnant. Once he was started on induction treatment along with abscess management, his symptoms improved significantly. During surgical exploration, the enterourachal fistula could not be identified and likely obliterated with the medical management. Timely initiation of CD management helped in reduction of bowel inflammation and resulted in limited surgical resection of only the ileocecal region. Similar to our patient, early aggressive management of $C D$ resulted in obliteration of fistula limiting extensive surgical resection in the 15-year-old male with $\mathrm{CD}$ described by Hollander et al. [8]. The MRI demonstrated an urachal abscess and suspected a fistula between the fluid collection and the adjacent inflamed bowel. He was aggressively treated with IV antibiotics and low dose steroids. During laparotomy, urachal abscess with adjacent phlegmon containing cecum and TI with no clear identifiable fistula was noted. This patient had resection of urachus with partial cystectomy and no resection of the bowel. Here the authors concluded that early medical management might have obliterated the small fistulous tract. Also in the patient reported by Mador and Blair [11], preoperative medical management with antibiotics and steroids reduced the inflammation considerably and ileocecectomy was carried out successfully using a laparoscopic approach.

Barthalomaues Cabrolius first described the persistence of the urachus in 1550 [16]. During fetal life, the urachus is a patent tubular structure that connects the allantois at the umbilicus to the dome of the urinary bladder residing in the space of Retzius [3]. Descent of the bladder into the pelvis, during the fourth-to-fifth month of gestation, stretches the urachus obliterating its lumen and forms the vestigial median umbilical ligament [11]. About 1-in-5,000 cases have incomplete obliteration resulting in various urachal abnormalities [17]. These abnormalities include (i) patent urachus, (ii) urachal cyst, sinus, or diverticulum, and (iii) atretic urachal remnant $[17,18]$. The urachal anomalies can be symptomatic, however in many instances they are incidentally found during abdominal imaging performed for other reasons. Common presenting symptoms in children with an urachal abnormality include suprapubic abdominal pain, dysuria, umbilical discharge, 
and periumbilical mass $[9,19]$. The majority of these symptoms occur as a result of infection. Another long term reported complication is malignant transformation within the urachal remnant [20]. Surgical resection usually is recommended for symptomatic urachal remnants. Management of asymptomatic urachal remnants remain controversial [20]. Although biological therapies such as anti-tumor necrosis factor alpha monoclonal antibody medications are recommended in the medical management of fistulizing $\mathrm{CD}$, the specific role of these therapies in $\mathrm{CD}$ with enterourachal fistulas is unknown due to paucity of information [11].

In summary, we report a case of $\mathrm{CD}$ complicated by an enterourachal fistula in which the initial diagnosis was delayed due to the non-specific presenting symptoms. Clinicians should have a high index of suspicion for this complication in CD patients presenting with symptoms suggestive of urachal anomalies (suprapubic abdominal pain, dysuria, umbilical discharge, periumbilical mass). Typical radiographic signs will help to confirm the diagnosis.

\section{REFERENCES}

1. Davidson ED. Crohn's disease with spontaneous cutaneous-urachovesicoenteric fistula. Dig Dis Sci 1980; 25:460-3.

2. Artigas JM, Blasco A, Mota J, Macho J, Gracia AI. Spontaneous enterourachocutaneous fistula in Crohn's disease: sonographic diagnosis. J Clin Ultrasound 1998;26:43-5.

3. Klineberg EO, James SP, Dunkin BJ. Crohn's disease complicated by a urachoenteric fistula. Dig Dis Sci 2002;47:1728-31.

4. Sugiyama Y, Kudo J, Tanaka J. Urachal abscess with Crohn's disease: a case report. Nishinihon J Urol 2003; 65:18-21.

5. Keir JA, McGregor R, Richards CJ, Windle R. An unusual presentation of Crohn's disease. Ann R Coll Surg Engl 2004;86:W22-3.

6. Bergman R, Sloots CE. [Diagnostic image (246). A man with faecal production of the umbilicus]. Ned Tijdschr Geneeskd 2005;149:1940. Dutch.

7. Weitten T, Coca C, Ben Abdelghani M, Rohr S, Boujan
E, Blicklé JF, et al. [A urachus cyst revealing a torpid Crohn's disease in a young adult with chronic fever]. Presse Med 2005;34:581-2. French.

8. Hollander LL, Girard ED, Ruscher KA, Sayej W, Kim C, Finck CM. Infected urachal cyst secondary to a Crohn's enterourachal fistula. J Pediatr Surg 2012;47: e43-6.

9. Yheulon CG, Derosa DC, Gagliano RA. Retained pill camera at an entero-uracho-vesical fistula site in a patient with Crohn's disease. Hawaii J Med Public Health 2013;72:186-9.

10. O'Brien D, Beatty N, Ramalanjaona G, Gress F, Deeb L. A rare case of septic urachal cyst mimicking abdominal fistualization in Crohn's disease [abstract]. Am J Gastroenterol 2013;108(Suppl 1):S417.

11. Mador BD, Blair GK. Pediatric Crohn disease complicated by an entero-uracho-cutaneous fistula. J Ped Surg Case Reports 2014;2:79-81.

12. Tsukui H, Koinuma K, Morimoto M, Horie H, Lefor AK, Kagaya Y, et al. Crohn's disease presenting as a ceco-urachal fistula. Clin J Gastroenterol 2017;10:32-6.

13. Solem CA, Loftus EV Jr, Tremaine WJ, Pemberton JH, Wolff BG, Sandborn WJ. Fistulas to the urinary system in Crohn's disease: clinical features and outcomes. Am J Gastroenterol 2002;97:2300-5.

14. Paşalega M, Calotă F, Paraliov T, Meşină C, Vîlcea D, Tomescu P, et al. [Crohn's disease. Clinical and therapeutical considerations]. Chirurgia (Bucur) 2005;100: 495-502. Romanian.

15. Ishii G, Tanaka N, Hara H, Ishii N, Matsumoto H. [Management of urinary complication in Crohn's disease]. Nihon Hinyokika Gakkai Zasshi 2007;98:757-63. Japanese.

16. RC Begg. The urachus: its anatomy, histology and development. J Anat 1930;64:170-83.

17. Blichert-Toft M, Koch F, Nielsen OV. Anatomic variants of the urachus related to clinical appearance and surgical treatment of urachal lesions. Surg Gynecol Obstet 1973;137:51-4.

18. Naiditch JA, Radhakrishnan J, Chin AC. Current diagnosis and management of urachal remnants. J Pediatr Surg 2013;48:2148-52.

19. Ashley RA, Inman BA, Routh JC, Rohlinger AL, Husmann DA, Kramer SA. Urachal anomalies: a longitudinal study of urachal remnants in children and adults. J Urol 2007;178:1615-8.

20. Gleason JM, Bowlin PR, Bagli DJ, Lorenzo AJ, Hassouna T, Koyle MA, et al. A comprehensive review of pediatric urachal anomalies and predictive analysis for adult urachal adenocarcinoma. J Urol 2015;193:632-6. 